\title{
Genetic Variability and Effect of Postflowering Drought on Stalk Sugar Content in Sorghum Mini Core Collection
}

\author{
Hari D. Upadhyaya,^ Sangam L. Dwivedi, Punna Ramu, Shailesh K. Singh, and Sube Singh
}

\begin{abstract}
Sweet sorghum [Sorghum bicolor (L.) Moench] is an important food, feed, and biofuel crop worldwide. Its stalks are rich in sugar, largely influenced by environments and crop stage when the stalks are harvested. This study evaluated sorghum mini core collection for stalk sugar content on the basis of Brix, phenology, and agronomic traits. Accessions were first classified into five groups based on flowering to match crop phenology, which together with controls were separately grown in split-plot design in vertisol under irrigated and drought stress conditions for two postrainy seasons. The main and interaction effects were significant for most of the traits. Drought stress significantly increased the mean Brix (12.11 to $26.76 \%$ ) in four of the five groups of accessions. The mean Brix increased under drought in 169 accessions, decreased in one accession, while the remaining accessions were not affected. IS 13294, 13549, 23216, 23684, 24139, 24939, and 24953 significantly recorded greater mean Brix (14.0 to $15.2 \%$ ) as compared with best control, IS 33844 (12.4\%), across environments. However, these accessions had lower yields and lower 100-seed weight. In contrast, IS 1004, 4698, 23891, and 28141 significantly outyielded IS 33844 by 11.7 to $22.7 \%$ and had almost the same Brix content $(\sim 13 \%)$. A hierarchical tree diagram grouped these accessions into two distinct clusters: accessions with significantly greater Brix but lower yield and those with high yield but with Brix similar to IS 33844 . The grouping also separated accessions largely on the basis of geographic regions and racial classification. The identified accessions are ideal resources for the development of cultivars with bioenergy traits.
\end{abstract}

International Crops Research Institute for the Semi-Arid Tropics (ICRISAT), Patancheru, Telangana, India. Received 17 Jan. 2014. ^Corresponding author (h.upadhyaya@cgiar.org).

Abbreviations: D, drought; DAP, days after planting; G, genotype; SCMR, SPAD chlorophyll meter reading; SSR, simple sequence repeat; $\mathrm{Y}$, year

$\mathrm{T}$ HE PRODUCTION OF BIOFUEL from plant-based biomass is becoming an attractive alternative to nonrenewable fossil energy sources. Ethanol as a substitute for gasoline is now being used in motor vehicles in many countries. The European Union has committed to increase the proportion of its renewable energy use from $9 \%$ in 2010 to $20 \%$ of total energy consumption by 2020 (EU, 2009). Similarly, the U.S. Energy Independence and Security Act of 2007 (Publ. L. 110-140, 19 Dec. 2007) is committed to derive up to $30 \%$ of transportation fuels from renewable sources, with an increase in the production of ethanol from 6400 million L in 1999 to 49,200 million L in 2010 (Renewable Fuels Association, 2012). China has set the target of producing 4 million $t$ of bioethanol in 2020 and 7.95 million t in 2030 (Bang et al., 2009b). The Government of India's policy of blending 5\% ethanol with petrol has generated demand for the cultivation of sweet sorghum as a potential bioenergy crop. In India and some other countries, the biofuel industry is promoting and commercializing sweet sorghum as an important feedstock for bioethanol production in the country (AgriFuels Limited, 2012; Praj Industries Limited, 2012; Tata Chemicals Limited, 2012).

Sorghum is a $\mathrm{C}_{4}$ multi-purpose crop with high photosynthetic efficiency, mostly grown for food, feed, and fuel. It is the sixth most important cereal after maize (Zea mays L.), rice (Oryza

Published in Crop Sci. 54:2120-2130 (2014).

doi: 10.2135/cropsci2014.01.0040

(C) Crop Science Society of America | 5585 Guilford Rd., Madison, WI 53711 USA

All rights reserved. No part of this periodical may be reproduced or transmitted in any form or by any means, electronic or mechanical, including photocopying, recording, or any information storage and retrieval system, without permission in writing from the publisher. Permission for printing and for reprinting the material contained herein has been obtained by the publisher. 
sativa L.), wheat (Triticum aestivum L.), barley (Hordeum vulgare L.), and oat (Avena sativa L.). On average, sorghum contributed $\sim 7 \%$ of $2448 \mathrm{~m} \mathrm{t}$ global cereals production in 2007 to 2011, with Africa and America contributing 38 to $39 \%$, respectively and Asia contributing 17\% (http:// www.faostat.fao.org, accessed 30 May 2013). Sweet sorghum belongs to the same species as grain sorghum, but is characterized by its rapid growth, sugar-rich stalk, and high biomass production, as well as its adaptation to adverse growing conditions (Reddy and Reddy, 2003; Reddy et al., 2005). Recently, sweet sorghum has gained importance as a potential bioenergy crop because of its high biomass and sugar production (Rooney et al., 2007), with a clear comparative advantage under dryland conditions where other leading bioethanol crops such as sugarcane (Saccharum officinarum L.) and maize cannot be grown (Tsuchihashi and Goto, 2008). Unlike maize, sweet sorghum does not compromise, but instead complements both food and energy security efforts (Gutjahr et al., 2013; Srinivasa Rao et al., 2009), grown for food, feed, and fuel that adapts to tropical, subtropical, and temperate zones. It is commercially grown as a bioenergy crop in the United States and European Union and is gaining importance in some countries in Africa, Asia, and the Mediterranean regions (Codesido et al., 2013; Kumar et al., 2011; Zegada-Lizarazu et al., 2010).

Genetic variability is the key to cultivar development. Biomass yield, juice yield, and sugar production are important bioenergy traits for ethanol production from sweet sorghum. Research at ICRISAT and in some other countries has shown sufficient genetic variation for stem sugar accumulation and associated traits among the limited sorghum germplasm and cultivars evaluated for bioenergy traits. For example, mean Brix (\%) (a measure of sugar, specifically sucrose content) among sorghum germplasm and/or cultivars ranged from 8 to $24 \%$ in India (Srinivasa Rao et al., 2009), 1 to 20\% in Japan (Kawahigashi et al., 2013), 5 to 23\% in Korea (Bang et al., 2009a; Choi et al., 2012), 6 to 20\% in southern Africa (Makanda et al., 2009, 2012), 10 to $19 \%$ in Spain (Codesido et al., 2013), and 6 to $17 \%$ in the United States (Graham et al., 2013). Likewise, large genetic variation in biomass has also been reported among sweet sorghum germplasm evaluated (e.g., 29 to $72 \mathrm{t} \mathrm{ha}^{-1}$ in tropical and 50 to $90 \mathrm{t} \mathrm{ha}^{-1}$ in temperate climate; Srinivasa Rao et al., 2009 and references cited therein). The environments and their interaction with genotypes have strong influence on the cultivar's adaptation. Limited research to date suggests significant genotype $\times$ environment interaction for traits related to high biomass production and stalk sugar content among the sweet sorghum germplasm lines (Makanda et al., 2012; Salazar et al., 2009; Srinivasa Rao et al., 2009; Zou et al., 2011). The phenological stage at which sorghum stalks are harvested, the planting time, the soil types, and the $\mathrm{N}$ fertilizer doses have significant influence on biomass and stalk sugar content (Almodares et al., 2013; Erickson et al., 2011; Makanda et al., 2009; Miri and Rana, 2012; Rao et al., 2013).

The total sugar in sorghum stalk juice is composed of sucrose, glucose, fructose, maltose, and xylose, with sucrose being the predominant sugar (Almodares et al., 2008; Prasad et al., 2007). Brix (\%) is commonly used to indicate total sugar content (Hagio et al., 1987), which is proportional to the total sugar and sucrose concentrations $(r=0.90$ and $r=0.89$, respectively) (Kawahigashi et al., 2013). Brix is not correlated with glucose or fructose and so neither the glucose nor the fructose concentrations in the sorghum juice is reflected by the Brix (Kawahigashi et al., 2013). In contrast, invert sugars are negatively correlated with sucrose content in sorghum (Almodares et al., 2008).

Worldwide, sorghum is largely grown during the rainy season in semiarid tropic areas of Africa and Asia, which is often characterized by increasing moisture stress as the season progresses. Both preflowering and postflowering drought stress in sorghum cause substantial losses to grain yield (Assefa et al., 2010; Blum, 2004; Kebede et al., 2001; Rosenow and Clark, 1995; Tuinstra et al., 1996). India is the largest producer of sorghum (around 3 million $t$ from 5.7 million ha) grown during the postrainy season (September or October to March or April), and severe drought is common as the crop in these areas relies largely on soil moisture stored during the preceding rainy season (Kholová et al., 2012).

Reduced germplasm subsets such as core (Frankel, 1984) and mini core (Upadhyaya and Ortiz, 2001) collections represent the diversity of the entire collection of a given species preserved in a genebank. They serve as the ideal genetic resources in identifying new sources of variation with agronomically beneficial traits (Upadhyaya et al., 2009a). This study was initiated to assess the genetic variation and effect of postflowering drought stress on stalk sugar content, phenology, and agronomic traits and to identify accessions with agronomically desirable traits, specifically for high stalk-sugar content, for use in crop improvement.

\section{MATERIALS AND METHODS}

Two hundred and forty two germplasm accessions of the sorghum mini core collection (Upadhyaya et al., 2009b), representing five races (caudatum 16.1\%, durra 12.4\%, guinea 12\%, kafir $8.7 \%$, bicolor $8.3 \%$ ) and 10 intermediate races (caudatum-bicolor 12.4\%; guinea-caudatum 11.2\%; durra-caudatum 7.9\%; durra-bicolor and kafir-caudatum each 2.9\%; kafir-durra 1.7\%; guinea-kafir 1.2\%; and guinea-bicolor, guinea-durra, and kafir-bicolor each 0.8\%) along with three controls (IS 2205, IS 18758, and IS 33844) were included in this study. IS 2205, a durra-bicolor landrace from India, is resistant to shoot fly and stem borer (Sharma, 1997). IS 18758, an advanced cultivar from Ethiopia characterized by yellow endosperm and resistance to anthracnose (Colletotrichum sublineolum Hann. Kabát et Bub.), belongs to guinea-caudatum race and was released as E 35-1 in Burkina Faso and Gambella 1107 in Burundi (Thakur 
et al., 2007). IS 33844, a durra landrace with pearly-white large grains and adapted to postrainy season, has been released as Parbhani Moti (SPV 1411) in India (Reddy et al., 2007).

The experiment was conducted at Patancheru, India $\left(78^{\circ} 12^{\prime} \mathrm{E}, 17^{\circ} 24^{\prime} \mathrm{N}\right.$, and $545 \mathrm{~m}$ asl) on vertisol Kasireddipally series-isohypothermic Typic Pellustert (El-Swaify et al., 1985) in a split-plot design in three replications using drought stress and control (fully irrigated) treatments as the main plot and genotypes as the subplot in five maturity groups (Group 1, flowered $<60 \mathrm{~d}$ after planting [DAP]; Group 2, flowered between 61 and 70 DAP; Group 3, flowered 71-80 DAP; Group 4, flowered 81-90 DAP; and Group 5, flowered >90 DAP] during the 2010 to 2011 and 2011 to 2012 postrainy seasons. The experiment was sown in the second week of October each year and harvested in April of the following years. The minimum monthly average temperature during postrainy seasons at Patancheru ranged from 10.9 to $22.8^{\circ} \mathrm{C}$, while the maximum monthly average temperature ranged from 27.3 to $37.5^{\circ} \mathrm{C}$. The cumulative rainfall during the crop season was $23.9 \mathrm{~mm}$ in 2010 to 2011 and $41.0 \mathrm{~mm}$ in 2011 to 2012 . The average daylength was 11.64 (11.08-12.75) hours each year. Plot size consisted of one row of $4 \mathrm{~m}$, with an inter- and intrarow spacing of 75 and 10 $\mathrm{cm}$, respectively. Seeds were sown at uniform depth, and cropspecific agronomic practices including plant protection were followed. Ammonium phosphate was applied at the rate of 150 $\mathrm{kg} \mathrm{ha}^{-1}$ as a basal dose, while urea was applied at the rate of 100 $\mathrm{kg} \mathrm{ha}^{-1}$ as top-dressing $3 \mathrm{wk}$ after planting. The fully irrigated plots received five irrigations (each time receiving about $7 \mathrm{~cm}$ water), while those in limited irrigation environment had one to four irrigations (on the basis of maturity groups). To stimulate drought-stressed environment, irrigation was stopped at 25 DAP in Group 1, 37 DAP in Group 2, 47 DAP in Group 3, 57 DAP in Group 4, and 66 DAP in Group 5. Data were recorded on Brix (\%), days to $50 \%$ flowering, plant height $(\mathrm{cm})$, head length and width $(\mathrm{cm})$, grain yield $\left(\mathrm{g} \mathrm{plant}^{-1}\right), 100$-seed weight (g), and SPAD chlorophyll meter reading (SCMR I) at 50\% flowering and $30 \mathrm{~d}$ after flowering (SCMR II). Days to 50\% flowering was recorded on per plot, while observations on plant height, head length, and head width on five plants were measured at maturity (IBPGR and ICRISAT, 1993). To measure the Brix (\%) (sucrose content) (Hagio et al., 1987), five plants were harvested at $40 \mathrm{~d}$ after flowering in each accession, and bulked stalks with leaves (but without the head) were crushed using three roller crushers (Jagdish no-6, Rajkot, India). The juice was then collected to measure Brix content. An Atago refractometer (digital refractometer PR-32 $\alpha$; ATAGO USA, Bellevue, WA) was used to measure Brix (\%). Data on two SCMR were recorded first at flowering (50\%) and later on at $30 \mathrm{~d}$ after flowering using SPAD-502 (Minolta Corp., Ramsey, NJ). Grain yield (g plant ${ }^{-1}$ ) was recorded on five representative plants. One hundred sound mature seeds were weighed to record observation on 100-seed weight $(\mathrm{g})$.

Analysis of variance was performed using split plot design in GenStat 14.1 software (VSN International, 2013) to derive the main (drought, year, and genotype) and interaction (drought $\times$ year, genotype $\times$ year, drought $\times$ genotype, drought $\times$ genotype $\times$ year) effects and their residuals. The mini core collection accessions (242) were part of the sorghum composite collection (3367 accessions), which was molecularly profiled using 41 polymorphic simple sequence repeats (SSRs) for dissecting population structure and diversity in composite collection (Billot et al., 2013). The previously generated $41 \mathrm{SSR}$ data points on the selected high Brix accessions (12) identified in this study were used to derive simple matching distance matrix for constructing a hierarchical tree diagram using DARwin 5.0 (Perrier et al., 2003).

\section{RESULTS}

Pooled analysis of variance showed that the main effects, namely drought, genotype, and year, were significant in all the five groups for Brix (except for drought in Group 1 and Group 5), days to 50\% flowering (except for drought in Group 4), plant height and head length (except for drought in Groups 2 and 4 and for drought and year in Group 5), head width (except for drought in Groups 2 and 4), and grain yield and 100-seed weight (except for drought in Group 5) (Table 1). Drought stress significantly affected SCMR I and SCMR II in Group 4 but not in the other groups, while both genotype and year effects were significant for SCMR I and SCMR II in all the five groups. In Group 1, significant drought $\times$ year interaction was observed for all traits except for days to $50 \%$ flowering. In the same group, genotype $\times$ year was observed for all traits except SCMR II, while drought $\times$ genotype was not observed for Brix and SCMR II. In Group 2, both drought $\times$ year and genotype $\times$ year were significant for all traits, while drought $\times$ genotype was not observed for SCMR II. In Group 3, genotype $\times$ year was significant for all the traits, while drought $\times$ year was not present for days to $50 \%$ flowering and SCMR II, and drought $\times$ genotype was not observed for SCMR I. In Group 4, genotype $\times$ year was significant for all traits. In this group, drought $\times$ year was not observed for Brix, head width, and SCMR II, while drought $\times$ genotype was absent for head length. In Group 5, genotype $\times$ year was not observed for SCMR I, while drought $\times$ year was not observed for days to $50 \%$ flowering, plant height, grain yield, and SCMR I and SCMR II. In the same group, drought $\times$ genotype was not present for 100-seed weight, SCMR I, and SCMR II. Drought $\times$ genotype $\times$ year interaction was not observed for head length, SCMR I, and SCMR II in Group 1; SCMR I and SCMR II in Group 2; SCMR I in Group 3; head length in Group 4; and for plant height, grain yield, SCMR I, and SCMR II in Group 5 (Table 1).

Drought stress in comparison to the irrigated control significantly increased the mean Brix in maturity Groups 1 to 4 , with percent increase ranging from 12.11 to $26.76 \%$ (Table 2). In Group 5, Brix was observed to be the same in both irrigated and drought-stressed conditions. However, the genotypes responded differently to drought stress for Brix in each group. For example, 16 accessions in Group 1, 67 in Group 2, 74 in Group 3, 16 in Group 4, and 2 in Group 5 had significantly greater Brix under drought stress condition than in irrigated control. The percent 
Table 1. Mean squares for Brix (\%), days to $50 \%$ flowering, plant height, head length and width, grain yield, $100-$ seed weight, and SPAD chlorophyll meter reading (SCMR) in sorghum mini core collection accessions evaluated for two postrainy seasons (2010-2011 and 2011-2012) under irrigated and drought-stressed conditions, Patancheru, India.

\begin{tabular}{|c|c|c|c|c|c|c|c|c|c|}
\hline $\begin{array}{l}\text { Source of } \\
\text { variation }\end{array}$ & $\begin{array}{l}\text { Brix } \\
\text { reading }\end{array}$ & $\begin{array}{c}\text { Days to } 50 \% \\
\text { flowering }\end{array}$ & $\begin{array}{l}\text { Plant } \\
\text { height }\end{array}$ & $\begin{array}{l}\text { Head } \\
\text { length }\end{array}$ & $\begin{array}{l}\text { Head } \\
\text { width }\end{array}$ & $\begin{array}{l}\text { Grain } \\
\text { yield }\end{array}$ & $\begin{array}{c}\text { 100-seed } \\
\text { weight }\end{array}$ & SCMR I & SCMR II \\
\hline & $\%$ & & +2 & $-\mathrm{cm}-$ & - & g plant ${ }^{-1}$ & $g$ & & \\
\hline \multicolumn{10}{|c|}{ Group 1 (36 entries including controls) } \\
\hline Replication & 5.4 & $19.0^{\star}$ & 507.0 & 0.2 & 1.8 & 10.7 & 0.1 & 45.0 & 330.6 \\
\hline Drought (D) & 152.7 & $64.4^{\star}$ & $94,883.6^{\star *}$ & $171.7^{\star}$ & $185.1^{*}$ & $2,418.1^{\star *}$ & $2.1^{\star \star}$ & $1,057.8$ & 946.7 \\
\hline Residual & 9.7 & 1.3 & 226.8 & 5.3 & 3.4 & 10.6 & 0.01 & 377.4 & 254.3 \\
\hline Genotype (G) & $22.9^{\star \star}$ & $576.0^{\star \star}$ & $15,400.7^{\star \star}$ & $327.2^{\star \star}$ & $324.5^{\star \star}$ & $657.8^{\star \star}$ & $4.1^{\star \star}$ & $221.6^{\star \star}$ & $274.7^{\star \star}$ \\
\hline$D \times G$ & 5.6 & $15.6^{\star \star}$ & $941.5^{\star \star}$ & $4.2^{\star}$ & $8.9^{\star \star}$ & $71.9^{\star \star}$ & $0.2^{\star *}$ & $36.7^{\star \star}$ & 25.2 \\
\hline Residual & 1.4 & 2.0 & 122.9 & 2.8 & 2.0 & 10.9 & 0.1 & 17.0 & 32.1 \\
\hline Year (Y) & $6.5^{\star}$ & $511.8^{\star *}$ & $34,537.2^{\star \star}$ & $94.9^{\star \star}$ & $86.7^{\star \star}$ & $2987.3^{\star \star}$ & $15.1^{\star \star}$ & $4365.5^{\star \star}$ & $682.9^{\star *}$ \\
\hline$D \times Y$ & $347.7^{\star \star}$ & 2.6 & $70,905.6^{\star \star}$ & $292.4^{\star \star}$ & $323.5^{\star \star}$ & $805.6^{\star \star}$ & $13.4^{\star \star}$ & $2840.3^{\star \star}$ & $1,274.3^{\star \star}$ \\
\hline$G \times Y$ & $9.5^{\star \star}$ & $72.4^{\star \star}$ & $818.4^{\star \star}$ & $17.6^{\star \star}$ & $13.1^{\star \star}$ & $71.0^{\star \star}$ & $0.5^{\star \star}$ & $39.4^{\star \star}$ & 41.3 \\
\hline$D \times G \times Y$ & $5.2^{\star \star}$ & $14.6^{\star \star}$ & $463.6^{* *}$ & 3.1 & $5.1^{\star \star}$ & $57.8^{\star \star}$ & $0.2^{* *}$ & 29.3 & 36.1 \\
\hline Residual & 1.2 & 2.5 & 114.9 & 3.5 & 1.8 & 12.5 & 0.1 & 19.8 & 35.5 \\
\hline \multicolumn{10}{|c|}{ Group 2 (85 entries including controls) } \\
\hline Replication & 5.2 & 29.4 & $2,530.8$ & 36.2 & 14.4 & 40.0 & 0.1 & 43.4 & $1,279.2$ \\
\hline Drought & $1,323.5^{\star \star}$ & $168.1^{\star \star}$ & $170,655.9^{*}$ & 78.2 & 49.1 & $3,874.4^{*}$ & $4.3^{* \star}$ & 71.0 & $2,268.5$ \\
\hline Residual & 6.4 & 4.3 & $3,353.9$ & 14.2 & 14.7 & 74.5 & 0.04 & 105.4 & 413.3 \\
\hline Genotype & $26.1^{\star *}$ & $298.8^{\star \star}$ & $13,644.3^{\star \star}$ & $375.9^{\star \star}$ & $155.7^{\star \star}$ & $523.6^{\star \star}$ & $6.2^{\star \star}$ & $216.1^{\star \star}$ & $229.1^{\star *}$ \\
\hline$D \times G$ & $5.0^{\star *}$ & $10.5^{\star \star}$ & $564.4^{\star \star}$ & $3.8^{\star *}$ & $10.5^{\star \star}$ & $58.6^{\star \star}$ & $0.1^{\star \star}$ & $26.0^{\star *}$ & 26.7 \\
\hline Residual & 1.5 & 2.3 & 152.6 & 2.5 & 1.9 & 12.5 & 0.04 & 13.2 & 21.1 \\
\hline Year & $74.3^{\star \star}$ & $3,718.7^{\star \star}$ & $18,603.9^{\star \star}$ & $252.1^{\star \star}$ & $380.2^{\star \star}$ & $672.5^{\star \star}$ & $47.7^{\star \star}$ & $11,710.0^{\star *}$ & $589.1^{\star *}$ \\
\hline$D \times Y$ & $619.2^{\star *}$ & $344.3^{\star \star}$ & $208,477.8^{\star \star}$ & $661.7^{\star \star}$ & $1,249.6^{\star \star}$ & $2,880.3^{\star \star}$ & $16.5^{\star \star}$ & $8,229.6^{\star \star}$ & $9,976.1^{\star *}$ \\
\hline$G \times Y$ & $7.6^{\star \star}$ & $55.0^{\star \star}$ & $569.5^{\star \star}$ & $11.7^{\star \star}$ & $14.4^{\star \star}$ & $69.8^{* *}$ & $0.3^{\star \star}$ & $46.4^{\star \star}$ & $67.3^{\star *}$ \\
\hline$D \times G \times Y$ & $3.5^{\star \star}$ & $14.7^{\star \star}$ & $400.6^{\star \star}$ & $5.9^{\star \star}$ & $18.5^{\star \star}$ & $63.9^{\star \star}$ & $0.2^{\star \star}$ & 17.3 & 26.2 \\
\hline Residual & 1.4 & 2.6 & 162.3 & 2.4 & 2.0 & 12.5 & 0.05 & 14.8 & 27.5 \\
\hline \multicolumn{10}{|c|}{ Group 3 (91 entries including controls) } \\
\hline Replication & 2.1 & 0.5 & $2,173.2$ & $20.5^{\star}$ & 4.6 & 58.9 & 0.01 & 44.2 & 84.1 \\
\hline Drought & $1,320.8^{\star \star}$ & $521.3^{\star *}$ & $63,489.7^{\star}$ & $26.1^{\star}$ & $198.6^{*}$ & $6,729 \cdot 3^{\star \star}$ & $1.0^{\star \star}$ & 26.8 & 599.3 \\
\hline Residual & 7.0 & 1.4 & $2,546.8$ & 0.5 & 4.2 & 27.7 & 0.02 & 92.5 & 140.7 \\
\hline Genotype & $30.2^{\star \star}$ & $270.7^{\star \star}$ & $14,493.4^{\star \star}$ & $695.8^{\star \star}$ & $251.4^{\star \star}$ & $679.6^{\star \star}$ & $8.1^{\star \star}$ & $185.9^{\star \star}$ & $232.7^{\star \star}$ \\
\hline$D \times G$ & $4.3^{\star *}$ & $11.6^{\star \star}$ & $687.6^{\star \star}$ & $4.1^{\star \star}$ & $12.6^{\star \star}$ & $61.3^{\star \star}$ & $0.1^{\star *}$ & 14.7 & $37.3^{\star *}$ \\
\hline Residual & 1.6 & 2.7 & 163.8 & 2.8 & 1.6 & 20.9 & 0.05 & 15.5 & 24.9 \\
\hline Year & $53.5^{\star \star}$ & $8,254.8^{\star *}$ & $369,940.7^{\star \star}$ & $279.7^{\star \star}$ & $186.2^{\star \star}$ & $1,066.5^{\star \star}$ & $22.5^{\star \star}$ & $11,256.8^{\star \star}$ & $4,495.5^{\star \star}$ \\
\hline$D \times Y$ & $329.5^{\star \star}$ & 3.5 & $16,078.4^{\star \star}$ & $371.6^{\star \star}$ & $470.4^{\star \star}$ & $2,266.9^{\star \star}$ & $11.9^{\star \star}$ & $1,872.9^{\star *}$ & 68.4 \\
\hline$G \times Y$ & $9.2^{\star \star}$ & $64.6^{\star}$ & $1,099.8^{\star \star}$ & $19.3^{\star *}$ & $43.2^{\star \star}$ & $84.6^{\star \star}$ & $0.3^{\star \star}$ & $47.1^{\star \star}$ & $77.5^{\star \star}$ \\
\hline$D \times G \times Y$ & $4.9^{* *}$ & $13.6^{\star}$ & $464.4^{\star \star}$ & $13.5^{\star \star}$ & $9.6^{\star \star}$ & $46.5^{\star \star}$ & $0.1^{\star \star}$ & 18.6 & $42.2^{\star \star}$ \\
\hline Residual & 1.4 & 2.5 & 143.3 & 2.7 & 1.3 & 14.3 & 0.04 & 15.6 & 19.1 \\
\hline \multicolumn{10}{|c|}{ Group 4 (34 entries including controls) } \\
\hline Replication & 2.8 & 3.3 & 114.2 & 6.3 & 29.5 & 6.3 & 0.01 & 33.0 & 7.3 \\
\hline Drought & $192.0^{\star \star}$ & 8.0 & $9,911.2^{*}$ & 13.9 & 36.8 & $1,136.9^{\star \star}$ & $1.0^{*}$ & $814.7^{\star \star}$ & $1,889.6^{\star}$ \\
\hline Residual & 0.5 & 1.7 & 78.0 & 9.2 & 9.0 & 2.6 & 0.03 & 7.4 & 37.9 \\
\hline Genotype & $24.4^{\star \star}$ & $512.9^{\star \star}$ & $25,185.1^{\star \star}$ & $470.5^{\star \star}$ & $248.4^{\star \star}$ & $833.0^{\star \star}$ & $4.4^{\star *}$ & $249.1^{\star \star}$ & $181.1^{\star \star}$ \\
\hline$D \times G$ & $5.0^{\star *}$ & $7.2^{\star \star}$ & $610.7^{\star \star}$ & 3.7 & $5.3^{\star \star}$ & $79.9^{\star *}$ & $0.1^{\star \star}$ & $33.5^{\star *}$ & $35.1^{\star}$ \\
\hline Residual & 1.3 & 2.6 & 159.6 & 3.4 & 2.3 & 18.0 & 0.03 & 13.3 & 22.2 \\
\hline Year & $138.4^{\star \star}$ & $4,430.4^{\star \star}$ & $139,427.7^{\star \star}$ & $64.1^{\star \star}$ & $340.5^{\star \star}$ & $1,509.2^{\star \star}$ & $0.7^{\star \star}$ & $2,157.2^{\star \star}$ & $4,988.3^{\star *}$ \\
\hline$D \times Y$ & 0.4 & $73.1^{\star \star}$ & $1,042 \cdot 3^{\star \star}$ & $21.4^{\star *}$ & 8.7 & $180.0^{\star \star}$ & $4.7^{\star \star}$ & $147.4^{\star \star}$ & 25.5 \\
\hline$G \times Y$ & $7.5^{\star \star}$ & $60.8^{\star \star}$ & $648.7^{\star \star}$ & $23.2^{\star \star}$ & $30.9^{\star \star}$ & $77.9^{\star \star}$ & $0.1^{\star \star}$ & $60.7^{\star \star}$ & $42.4^{\star *}$ \\
\hline$D \times G \times Y$ & $3.6^{\star \star}$ & $9.7^{\star *}$ & $421.4^{\star \star}$ & 4.4 & $4.9^{\star \star}$ & $48.9^{\star \star}$ & $0.1^{\star \star}$ & $31.8^{\star *}$ & $33.7^{\star}$ \\
\hline Residual & 1.5 & 2.5 & 147.2 & 3.2 & 2.6 & 15.2 & 0.03 & 11.6 & 19.2 \\
\hline \multicolumn{10}{|c|}{ Group 5 (11 entries including controls) } \\
\hline Replication & 3.0 & 7.2 & 404.6 & 0.4 & 2.2 & 224.1 & 0.01 & 53.2 & 43.8 \\
\hline Drought & 6.9 & $40.3^{\star *}$ & $7,445.8^{\star \star}$ & 14.6 & $76.7^{\star \star}$ & $952.9^{*}$ & 0.003 & 97.8 & 263.3 \\
\hline Residual & 5.4 & 0.8 & 43.6 & 2.5 & 0.4 & 18.9 & 0.002 & 8.9 & 18.5 \\
\hline
\end{tabular}


Table 1. Continued.

\begin{tabular}{|c|c|c|c|c|c|c|c|c|c|}
\hline $\begin{array}{l}\text { Source of } \\
\text { variation }\end{array}$ & $\begin{array}{c}\text { Brix } \\
\text { reading }\end{array}$ & $\begin{array}{c}\text { Days to } 50 \% \\
\text { flowering }\end{array}$ & $\begin{array}{l}\text { Plant } \\
\text { height }\end{array}$ & $\begin{array}{l}\text { Head } \\
\text { length }\end{array}$ & $\begin{array}{l}\text { Head } \\
\text { width }\end{array}$ & $\begin{array}{l}\text { Grain } \\
\text { yield }\end{array}$ & $\begin{array}{c}100 \text {-seed } \\
\text { weight }\end{array}$ & SCMR I & SCMR II \\
\hline Genotype & $45.6^{\star *}$ & $1,474.6^{\star *}$ & $14,110.5^{\text {** }}$ & $341.6^{\star *}$ & $243.2^{\star * *}$ & $873.1^{\star \star *}$ & $3.8^{* \star}$ & $197.5^{\star \star}$ & $132.6^{\star *}$ \\
\hline$D \times G$ & $7.1^{\star \star}$ & $23.9^{* \star}$ & $376.4^{*}$ & $7.8^{\star \star}$ & $21.8^{\star *}$ & $147.7^{\star \star}$ & 0.05 & 33.3 & 33.6 \\
\hline Residual & 2.8 & 1.8 & 181.1 & 2.4 & 1.3 & 43.8 & 0.04 & 18.1 & 22.6 \\
\hline Year & $12.6^{\star *}$ & $3,800.1^{\star *}$ & $105,201.6^{\star *}$ & 0.5 & $199.5^{\star \star}$ & $2247.3^{\star *}$ & $1.4^{\star *}$ & $1,150.2^{\star *}$ & $4,446.1^{\star \star}$ \\
\hline$D \times Y$ & $12.8^{* *}$ & 12.6 & 0.3 & $81.5^{\star \star}$ & $172.1^{\star \star}$ & 30.4 & $2.6^{\star *}$ & 26.1 & 73.8 \\
\hline$G \times Y$ & $4.8^{* *}$ & $65.6^{\star \star}$ & $773.3^{\star \star}$ & $16.3^{\star \star}$ & $44.8^{\star \star}$ & $163.2^{\star *}$ & $0.2^{* \star}$ & 8.6 & $53.8^{*}$ \\
\hline$D \times G \times Y$ & $2.8^{*}$ & $10.9^{* *}$ & 176.3 & $7.2^{\star *}$ & $34.8^{* *}$ & 46.8 & $0.1^{* \star}$ & 9.3 & 45.8 \\
\hline Residual & 1.3 & 3.4 & 175.3 & 2.4 & 2.4 & 21.5 & 0.03 & 16.0 & 18.9 \\
\hline
\end{tabular}

* Significant at the 0.05 probability level.

** Significant at the 0.01 probability level.

Table 2. Effect of drought on Brix (\%) in five maturity groups of sorghum mini core collection accessions evaluated for two postrainy seasons (2010-2011 and 2011-2012) under irrigated and drought-stressed conditions, Patancheru, India.

\begin{tabular}{|c|c|c|c|c|}
\hline \multirow[b]{2}{*}{ Category } & \multirow[b]{2}{*}{$\begin{array}{c}\text { No. } \\
\text { entries }\end{array}$} & \multicolumn{2}{|c|}{ Mean Brix ${ }^{\dagger}$} & \multirow{2}{*}{$\begin{array}{c}\% \text { increase } \\
\text { in Brix under } \\
\text { drought } \\
\text { stress }\end{array}$} \\
\hline & & Irrigated & $\begin{array}{l}\text { Drought } \\
\text { stress }\end{array}$ & \\
\hline \multicolumn{5}{|c|}{$\%$} \\
\hline Group 1 & 36 & $8.70 \mathrm{~b}$ & $9.86 a$ & 13.33 \\
\hline Group 2 & 85 & $8.78 b$ & $11.13 a$ & 26.76 \\
\hline Group 3 & 91 & $9.87 \mathrm{~b}$ & $12.10 a$ & 22.59 \\
\hline Group 4 & 34 & $11.48 b$ & $12.87 a$ & 12.11 \\
\hline Group 5 & 11 & $12.67 a$ & $13.76 a$ & 8.60 \\
\hline
\end{tabular}

${ }^{\dagger}$ Means were tested by Newman-Keuls test (Newman, 1939; Keuls, 1952); means followed by different letters differ significantly at $p=0.05$

increase in Brix on these accessions ranged from 14.4 to $51.5 \%$ in Group 1, 14.7 to $70.2 \%$ in Group 2, 11.2 to $65.7 \%$ in Group 3, 12.0 to $52.8 \%$ in Group 4, and 14.9 to $34.4 \%$ in Group 5 (data not given). Drought stress condition, in comparison to irrigated control, significantly reduced Brix in IS 13549 in Group 4. Drought in the remaining accessions had no or only marginal effect on Brix.

IS 33844 (also known as Parbhani Moti or SPV1411), one of the three controls, is the most popular sorghum cultivar in India, widely grown during rabi (postrainy) season (Reddy et al., 2007). Seven accessions; IS 23216 in Group 3; IS 13294, 13549, 23684, 24939, and 24953 in Group 4; and IS 24139 in Group 5, had significantly greater Brix (14.1-15.2\%) than the control IS 33844 (11.3 to $12.2 \%)$ (Table 3 ). However, these accessions yielded poorly (6.8 to $17.1 \mathrm{~g} \mathrm{plant}^{-1}$ ) and had much lower 100-seed weight (1.6 to $2.6 \mathrm{~g}$ ) than IS 33844 (which had grain yield of 36.0 to 42.4 g plant ${ }^{-1}$ and 100 -seed weight of 3.5 to $3.6 \mathrm{~g}$ ). In contrast, four accessions, IS 4698 in Group 1, IS 1004 and IS 23891 in Group 2, and IS 28141 in Group 3, had significantly more yield (11.7\% to $22.7 \%)$ and comparable Brix (11.7 to $12.8 \%)$ and 100 -seed weight $(3.2$ to $5.2 \mathrm{~g}$ ) as compared with IS 33844 (Brix, 12.2 to 13.4\%; 100-seed weight, 3.4 to $3.6 \mathrm{~g})$. IS 4698,23891 , and 28141 were comparable to IS 33844 in terms of days to $50 \%$ flowering ( $77 \mathrm{~d}$ ), while
IS 1004, 13294, 13549, 23684, 24939, 24953, and 24139 flowered 4 to $24 \mathrm{~d}$ later than IS 33844 . For plant height, IS 1004, 23891, 13294, 13549, and 24139 were taller than IS 33844 (234 to 269 cm). For SCMR, IS 23891 in Group 2 and IS 23216 and 28141 in Group 3 had significantly greater SCMR, both at flowering and $30 \mathrm{~d}$ after flowering, than IS 33844. Similarly, some of the high Brix accessions also had significantly greater head length and head width, like IS 23684 in Group 4 and IS 24139 in Group 5 (Table 3).

Plant height (except for Group 4) and days to 50\% flowering in irrigated and drought stress environments were significantly and positively correlated with Brix (Table 4). Grain yield was positively and significantly associated with Brix in irrigated and drought-stress environments in Group 1, while it was significantly negatively associated with Brix in Group 4. SCMR I (at flowering) under drought stress environments was negatively correlated with Brix in all the five groups. However, no such association was discovered (except for negative association in Group 3) between Brix and SCMR II (30 d after flowering) under drought stress environments. Head length, head width, and 100-seed weight were positively correlated $(r=0.72, \mathrm{p}<0.01)$ with Brix under drought stress environments in Group 5 (Table 4).

This study identified seven sorghum accessions with significantly greater Brix content than the control, IS 33844 , but with lower yields. Likewise, four accessions significantly outyielded the control, IS 33844, but with similar Brix content. A hierarchical tree diagram based on Unweighted Pair Group Method with Arithmetic Mean and simple matching dissimilarity matrix involving these 12 accessions including IS 33844 (which ranged from 0.342 to 0.859 among 66 comparison pairs based on 41 SSR data points on 12 genotypes) grouped the accessions into two distinct clusters (Fig. 1). The high-yielding accessions with Brix comparable to the controls sided with IS 33844 in Cluster 1, while those low-yielding but with significantly greater Brix than IS 33844 grouped separately in Cluster 2. The two clusters separated at dissimilarity of $\sim 0.28$. 
Table 3. Performance of selected sorghum mini core accessions for Brix (\%), grain yield, days to $50 \%$ flowering, plant height, head length and width, 100-seed weight, and SPAD chlorophyll meter reading (SCMR) evaluated for two postrainy seasons (2010-2011 and 2011-2012), Patancheru, India.

\begin{tabular}{|c|c|c|c|c|c|c|c|c|c|}
\hline & Brix & $\begin{array}{l}\text { Grain } \\
\text { yield }\end{array}$ & $\begin{array}{l}\text { Days to } 50 \% \\
\text { flowering }\end{array}$ & $\begin{array}{l}\text { Plant } \\
\text { height }\end{array}$ & $\begin{array}{l}\text { Head } \\
\text { length }\end{array}$ & $\begin{array}{l}\text { Head } \\
\text { width }\end{array}$ & $\begin{array}{c}\text { 100-seed } \\
\text { weight }\end{array}$ & SCMR I & SCMR II \\
\hline & $\%$ & $\mathrm{~g} \mathrm{plant}^{-1}$ & & 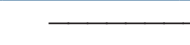 & $\mathrm{cm}$ & - & g & & \\
\hline \multicolumn{10}{|l|}{ Group 1} \\
\hline IS 4698 & 12.8 & $43.3^{\star}$ & 77.0 & 201.2 & 13.9 & 6.3 & 3.2 & 40.4 & 31.9 \\
\hline \multicolumn{10}{|l|}{ Control } \\
\hline IS 33844 & 13.4 & 35.3 & 77.6 & 213.3 & 14.1 & 6.5 & 3.4 & 40.2 & 31.4 \\
\hline $\begin{array}{l}\text { Trial mean } \\
\text { (range) }\end{array}$ & $\begin{array}{c}9.5 \\
(7.8-13.5)\end{array}$ & $\begin{array}{c}19.4 \\
(9.1-43.3)\end{array}$ & $\begin{array}{c}63.1 \\
(51.5-80.3)\end{array}$ & $\begin{array}{c}182.5 \\
(115.8-250.3)\end{array}$ & $\begin{array}{c}22.4 \\
(13.9-36.6)\end{array}$ & $\begin{array}{c}9.1 \\
(4.4-6.6)\end{array}$ & $\begin{array}{c}2.6 \\
(1.6-4.3)\end{array}$ & $\begin{array}{c}47.4 \\
(36.7-56.3)\end{array}$ & $\begin{array}{c}33.7 \\
(26.1-46.0)\end{array}$ \\
\hline LSD (5\%) & 0.9 & 2.6 & 1.1 & 9.0 & 1.3 & 1.1 & 0.2 & 3.1 & 4.4 \\
\hline CV (\%) & 14.8 & 23.7 & 3.5 & 7.4 & 8.1 & 17.4 & 10.5 & 9.6 & 17.4 \\
\hline \multicolumn{10}{|l|}{ Group 2} \\
\hline IS 1004 & 12.3 & $38.7^{*}$ & 85.8 & 261.7 & 9.1 & 5.8 & 3.8 & 41.5 & 31.8 \\
\hline IS 23891 & 11.7 & $40.9^{\star}$ & 75.6 & 250.4 & $16.8^{*}$ & 8.7 & $5.1^{*}$ & $47.1^{\star}$ & $40.3^{*}$ \\
\hline \multicolumn{10}{|l|}{ Control } \\
\hline IS 33844 & 13.1 & 33.5 & 77.3 & 234.5 & 14.7 & 6.9 & 3.6 & 41.4 & 32.8 \\
\hline $\begin{array}{l}\text { Trial mean } \\
\text { (range) }\end{array}$ & $\begin{array}{c}10.0 \\
(7.5-13.9)\end{array}$ & $\begin{array}{c}21.3 \\
(10.3-40.9)\end{array}$ & $\begin{array}{c}68.0 \\
(52.5-85.8)\end{array}$ & $\begin{array}{c}260.8 \\
(130.7-280.0)\end{array}$ & $\begin{array}{c}20.6 \\
(6.0-36.1)\end{array}$ & $\begin{array}{c}8.9 \\
(5.0-9.6)\end{array}$ & $\begin{array}{c}2.4 \\
(1.3-5.1)\end{array}$ & $\begin{array}{c}46.7 \\
(36.3-55.2)\end{array}$ & $\begin{array}{c}33.2 \\
(24.5-44.8)\end{array}$ \\
\hline LSD (\%) & 1.0 & 2.9 & 1.2 & 9.5 & 1.3 & 1.0 & 0.2 & 2.9 & 3.6 \\
\hline CV (\%) & 13.5 & 22.2 & 3.3 & 7.0 & 8.5 & 25.8 & 11.0 & 8.4 & 15.7 \\
\hline \multicolumn{10}{|l|}{ Group 3} \\
\hline IS 23216 & $14.7^{\star}$ & 12.3 & 85.6 & 243.9 & 16.1 & 6.5 & 1.9 & $48.4^{\star}$ & $38.4^{\star}$ \\
\hline IS 28141 & 11.8 & $40.2^{*}$ & 75.7 & 242.5 & 9.7 & 6.5 & $5.2^{*}$ & $49.8^{*}$ & $42.7^{\star}$ \\
\hline \multicolumn{10}{|l|}{ Control } \\
\hline IS 33844 & 12.2 & 36.0 & 77.7 & 254.5 & 15.0 & 7.0 & 3.5 & 43.7 & 29.5 \\
\hline $\begin{array}{l}\text { Trial mean } \\
\text { (range) }\end{array}$ & $\begin{array}{c}11.0 \\
(7.8-14.7)\end{array}$ & $\begin{array}{c}23.1 \\
(0.3-40.2)\end{array}$ & $\begin{array}{c}75.8 \\
(66.5-95.1)\end{array}$ & $\begin{array}{c}238.8 \\
(104.4-323.0)\end{array}$ & $\begin{array}{c}21.8 \\
(6.8-47.0)\end{array}$ & $\begin{array}{c}8.7 \\
(4.1-0.9)\end{array}$ & $\begin{array}{c}2.4 \\
(0.4-5.2)\end{array}$ & $\begin{array}{c}45.7 \\
(38.1-55.2)\end{array}$ & $\begin{array}{c}32.5 \\
(17.7-43.9)\end{array}$ \\
\hline LSD (5\%) & 1.0 & 3.8 & 1.2 & 9.8 & & & 0.2 & & \\
\hline CV (\%) & 12.7 & 19.7 & 2.8 & 6.1 & & & 9.9 & & \\
\hline \multicolumn{10}{|l|}{ Group 4} \\
\hline IS 13294 & $14.5^{\star}$ & 13.0 & 101.0 & 289.1 & $25.0^{*}$ & 7.4 & 1.8 & 36.1 & 28.3 \\
\hline IS 13549 & $14.0^{*}$ & 15.9 & 87.2 & 299.8 & 16.6 & $9.4^{*}$ & 1.8 & 42.3 & 27.9 \\
\hline IS 23684 & $14.5^{*}$ & 6.8 & 96.5 & 273.8 & $23.9^{*}$ & $10.4^{\star}$ & 1.6 & 32.1 & 27.2 \\
\hline IS 24939 & $14.6^{\star}$ & 12.2 & 84.8 & 233.3 & $18.8^{*}$ & 7.3 & 1.8 & 40.1 & 31.0 \\
\hline IS 24953 & $14.1^{*}$ & 17.1 & 80.8 & 247.0 & $21.4^{*}$ & 7.4 & 2.6 & 44.0 & 34.0 \\
\hline \multicolumn{10}{|l|}{ Control } \\
\hline IS 33844 & 11.3 & 40.8 & 77.5 & 269.0 & 15.5 & 6.4 & 3.6 & 49.3 & 31.7 \\
\hline $\begin{array}{l}\text { Trial mean } \\
\text { (range) }\end{array}$ & $\begin{array}{c}12.1 \\
(8.9-14.6)\end{array}$ & $\begin{array}{c}22.2 \\
(5.8-42.8)\end{array}$ & $\begin{array}{c}84.9 \\
(70.3-101.1)\end{array}$ & $\begin{array}{c}265.8 \\
(152.3-357.9)\end{array}$ & $\begin{array}{c}21.9 \\
(8.7-35.2)\end{array}$ & $\begin{array}{c}8.9 \\
(5.1-4.4)\end{array}$ & $\begin{array}{c}2.2 \\
(1.0-3.9)\end{array}$ & $\begin{array}{c}42.7 \\
(32.1-53.5)\end{array}$ & $\begin{array}{c}31.8 \\
(26.3-44.9)\end{array}$ \\
\hline LSD (5\%) & 0.9 & 3.3 & 1.2 & 9.9 & 1.5 & & 0.1 & 2.6 & 3.6 \\
\hline CV (\%) & 11.3 & 20.7 & 2.3 & 5.4 & 8.5 & & 9.9 & 9.2 & 14.9 \\
\hline \multicolumn{10}{|l|}{ Group 5} \\
\hline IS 24139 & $15.2^{*}$ & 13.8 & 95.0 & 301.6 & $31.7^{\star}$ & $14.7^{\star}$ & 1.7 & 38.8 & 31.2 \\
\hline \multicolumn{10}{|l|}{ Control } \\
\hline IS 33844 & 12.2 & 42.4 & 77.3 & 262.7 & 15.0 & 6.6 & 3.5 & 46.6 & 32.2 \\
\hline $\begin{array}{l}\text { Trial mean } \\
\text { (range) }\end{array}$ & $\begin{array}{c}12.7 \\
(8.0-15.2)\end{array}$ & $\begin{array}{c}21.6 \\
(3.8-42.4)\end{array}$ & $\begin{array}{c}91.5 \\
(69.3-118.6)\end{array}$ & $\begin{array}{c}253.5 \\
(170.1-301.6)\end{array}$ & $\begin{array}{c}23.8 \\
(15.0-32.7)\end{array}$ & $\begin{array}{c}9.7 \\
(5.7-9.2)\end{array}$ & $\begin{array}{c}2.2 \\
(1.4-3.5)\end{array}$ & $\begin{array}{c}41.7 \\
(37.0-47.6)\end{array}$ & $\begin{array}{c}31.1 \\
(27.9-33.8)\end{array}$ \\
\hline LSD (5\%) & 1.4 & 5.4 & 1.1 & 10.8 & 1.2 & 0.8 & 0.1 & 3.1 & 4.2 \\
\hline CV (\%) & 10.1 & 22.9 & 2.3 & 4.9 & 7.2 & 29.2 & 9.8 & 9.4 & 16.8 \\
\hline
\end{tabular}

* Significant at the 0.05 probability level.

\section{DISCUSSION}

Sorghum is now recognized as one of the most important food, feed, and bioenergy crops worldwide, mostly grown in semiarid and temperate regions. Sorghum is a $\mathrm{C}_{4}$ plant characterized by high photosynthetic- and radiation-use efficiency. It is more tolerant to drought, salinity, and heat stress than other cereal crops (Schittenhelm and Schroetter, 2014; Tari et al., 2013). Sweet sorghum, in comparison to sugarcane, is better suited for the production of ethanol because it requires one-fourth the amount of water that 
sugarcane needs, has more total sugars in the juice of the mature plant than sugarcane, and the ethanol is sulfur-free and cleaner than molasses-based ethanol. More importantly, sweet sorghum can be harvested in 3 to 4 mo (10-12 mo in the case of sugarcane). It is adapted to subtropical, tropical, and temperate climates; is resistant to drought, salinity, and water logging and grows well in marginal lands; and produces high biomass and grain yields, with no or relatively less food-feed-bioenergy tradeoff (Almodares and Hadi, 2009; Rooney et al., 2007; Srinivasa Rao et al., 2009; Tsuchihashi and Goto, 2008). The sugar content in the sweet sorghum stalk juice accumulates mostly before anthesis, varies little among sowing dates or between anthesis and maturity (Gutjahr et al., 2013), and contains sucrose and invert sugars (glucose, fructose, maltose, and xylose) which could be easily converted to ethanol (Almodares et al., 2008). Stem sugar content in sorghum is closely correlated with Brix, indicating its value as an index of sugar in stem juice (Tsuchihashi, 2004).

Both preflowering and postflowering drought stress have been reported to cause substantial losses to grain and stover yields in cereals, including sorghum (Assefa et al., 2010; Dwivedi et al., 2010; Tari et al., 2013). Stalk sugar content is one of the important traits (days to flowering, plant height, stalk weight, juice volume, and juice weight) differentiating sweet sorghum from those of grain types.

Conflicting reports are available about the effect of drought stress on stalk sugar content in sorghum. Some reports indicate that sugar accumulation is not sensitive to drought (Massacci et al., 1996), while others say delayed irrigation (at $21 \mathrm{~d}$ in comparison to 7 and $14 \mathrm{~d}$ ) significantly decreases sucrose content (Almodares et al., 2013). These conclusions, however, were mostly drawn from one season experiment involving few germplasm lines. It is also possible that the drought stress treatment in these previous reports was not enough to detect major differences in stalk sugar content between the drought stress and the irrigated control. Nutritional traits are highly affected by genotype, environment, and genotype $\times$ environment interaction effects (Dwivedi et al., 2013). It is therefore necessary to screen a subset of germplasm that represents the variability present in the entire collection of a given species in a genebank, and in multiple locations (or at least two seasons in one location) under defined drought stress conditions (pre- or postflowering drought) to quantify the effect of drought stress on stalk sugar content in sorghum.

The mini core collection of sorghum (242 accessions and three controls), categorized into five groups on the basis of phenology (flowering), was evaluated for stalk sugar content (as measured by Brix \%) and selected agronomic traits (days to $50 \%$ flowering, plant height, head length and width, grain yield plant ${ }^{-1}$, 100-seed weight, and SCMR) for two postrainy season environments under irrigated and drought stress conditions. The main effects 


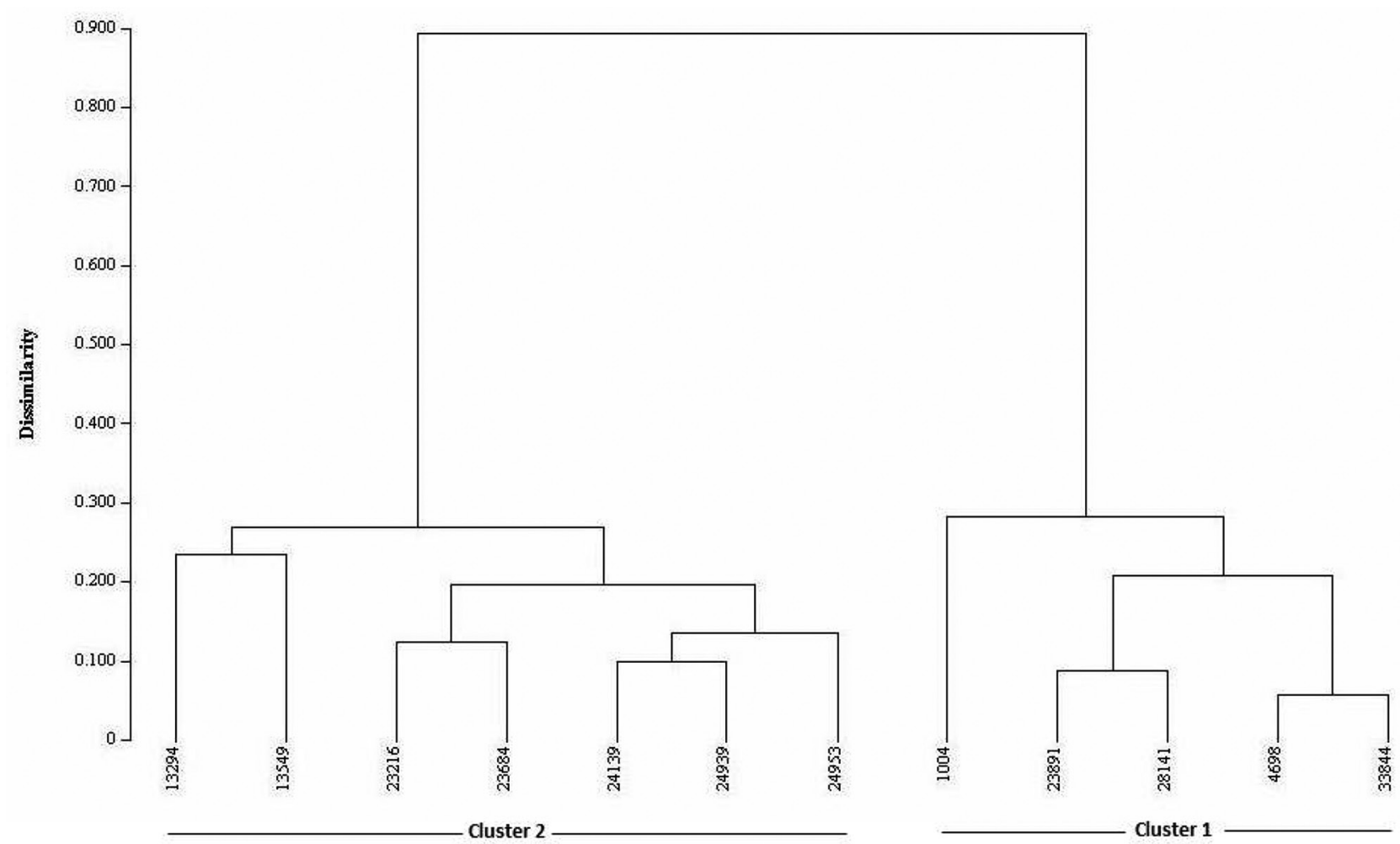

Figure 1. A hierarchical tree diagram of 11 selected sweet sorghum accessions and a control cultivar IS 33844 based on simple matching dissimilarity of 41 SSR markers. Cluster 1 accessions include those that significantly outyielded but with similar Brix to that of control, IS 33844, while cluster II accessions include those with low yields but with significantly greater Brix than IS 33844.

(drought, year, and genotype), as well as the two-way (drought $\times$ genotype, drought $\times$ year, and year $\times$ genotype) and the three-way (drought $\times$ genotype $x$ year) interaction effects were significant for most of the traits, including Brix (Table 1). A previous study involving sweet sorghum hybrids, R-lines, and varieties evaluated in rainy and postrainy seasons at Patancheru, India also reported significant genotype $\times$ season effect for total soluble sugar and sugar yield (Srinivasa Rao et al., 2009). Drought stress, in comparison to irrigated control, significantly increased Brix, and it varied from 8.60 to $26.76 \%$ (13.33\% in Group $1,26.76 \%$ in Group 2, 22.59\% in Group 3, $12.11 \%$ in Group 4, and 8.60\% in Group 5) (Table 2). Genotypes responded differently for Brix under drought-stress conditions. For example, 16 accessions each in Groups 1 and 4, 67 in Group 2, 74 in Group 3, and 2 in Group 5 had significantly greater Brix under drought stress conditions, while IS 13549 in Group 4 had significantly lower Brix. Drought stress in some other accessions had no significant effect on Brix in each group.

Sugars have long been known to increase in a wide range of plants grown at low moisture levels. For example, increased sugar content in roots and/or leaves was observed in drought-stressed chicory (Cichorium intybus L.), barley, cotton (Gossypium hirsutum L.), maize, sorghum, and wheat (de Roover et al., 2000; Eaton and Ergle, 1948; Kameli and Lösel, 1993; Massacci et al., 1996; Méndez et al., 2011; Mohammadkhani and Heidari,
2008; Vassiliev and Vassiliev, 1936). However, the rate and extent of increase in sugar content may depend on the environmental conditions, species, and genotypes within the species. Further, quantitative studies of biochemical and physiological traits in Arabidopsis thaliana (L.) Heynh. under stress revealed that prestress sugar concentrations were correlated with subsequent stress tolerance (Ramel et al., 2009). More recently, comparative metabolite profiling of drought-stressed versus control (irrigated) plants revealed that the stress substantially alters 143 metabolite compounds with highly reproducing patterns. The majority of the sugars (mannose, glucose, isomaltose, fructose, and sucrose in leaves and glucose, fructose, sucrose, trehalose, and mannitol in roots) significantly increased on stress in both leaves and roots of sorghum (Pavli et al., 2013). In the present study, drought stress significantly increased Brix in most of the accessions in each group. However, there were few accessions in each group that had similar Brix under irrigated and drought stress conditions. It is known that sugars impart stress tolerance (Pavli et al., 2013; Ramel et al., 2009), and this study will contribute further to validating that premise.

Agronomically, four accessions (IS 4698 in Group 1, IS 1004 and 23891 in Group 2, and IS 28141 in Group 3) significantly outyielded the best control IS 33844 (33.5 to $36.0 \mathrm{~g} \mathrm{plant}^{-1}$ ) by 11.7 to $22.7 \%$. However, these accessions had Brix similar to that of the control (Brix, 13\%). A previous study also reported some germplasm with high 
grain yield and high stalk sugar content in sorghum (Gutjahr et al., 2013). Seven accessions (IS 23216 in Group 3; IS 13294, 13549, 23684, 24939, 24953 in Group 4; IS 24139 in Group 5) showed significantly greater Brix (14 to 15\%) than the control (Brix, 13\%). The accessions with high Brix were however found to have lower yield (6.8-17.1 g plant $^{-1}$ ) and 100-seed weight (1.6-2.6 g), and flowered later (9-25 d) than the control IS 33844, which flowered in 77 to $81 \mathrm{~d}$, yielded 33 to $42 \mathrm{~g} \mathrm{plant}^{-1}$ grains, and had 100-seed weight of 3.4 to $3.6 \mathrm{~g}$ (Table 3). IS 13294, 13549, and 24139 had significantly greater plant height and head length, while IS 23684 had comparable plant height but significantly greater head length and head width than the control IS 33844. Previous studies have shown sufficient genetic variation for Brix among sorghum germplasm and/ or cultivars evaluated, with few accessions having high Brix (Almodares and Hadi, 2009; Bang et al., 2009a,b; Choi et al., 2012; Codesido et al., 2013; Makanda et al., 2012; Reddy et al., 2005; Salazar et al., 2009; Srinivasa Rao et al., 2009; Zou et al., 2011). Research at ICRISAT and in the United States have shown that it is possible to combine high grain yield and high stalk sugar content into improved genetic background in sorghum (Murray et al., 2008; Reddy et al., 2005; Srinivasa Rao et al., 2009).

Significant positive and moderate associations involving Brix with days to $50 \%$ flowering and plant height under both irrigated and drought stress conditions (Table 4), which support to previous reports (Murray et al., 2008; Srinivasa Rao et al., 2009; Zou et al., 2011), revealed that these two traits confer greater advantage to high Brix and should be considered in breeding programs to develop cultivars with greater Brix content in sorghum.

The genetic dissimilarity among 66 pair-wise comparisons involving 41 SSR data points on 12 accessions with significantly greater or similar Brix to that of IS 33844 ranged from 0.342 to 0.859 , and a hierarchical tree diagram grouped these accessions into two distinct clusters. Cluster 1 consists of high-yielding accessions with Brix comparable to the control, IS 33844. Cluster 2 consists of accessions that showed significantly greater Brix than IS 33844 but have lower yield (Fig. 1). The grouping clearly separated accessions into distinct geographical diversity. For example, accessions from south and west Asia grouped together in Cluster 1, while those from South America and Central America sided with accessions from eastern Africa in Cluster 2. This separation of accessions into two distinct clusters also reflected racial diversity. For example, durra or durra-caudatum types grouped together in Cluster 1, whereas those with guinea, bicolor, guinea-caudatum, and caudatum-bicolor types grouped in Cluster 2. IS 33884 is known for its large-size pearl-shaped grains and was released for rabi-season (October to March) cultivation in India. IS 23684, 24939, and 24953 were reported resistant to anthracnose and leaf blight [Exserohilum turcicum (Pass.)
K.J. Leonard \& Suggs] (Sharma et al., 2012), while IS 13294 and 13549 were reported resistant to maize dwarf mosaic virus (Seifers et al., 2012). In terms of susceptibility to insect pests, research at ICRISAT has shown that while most of these accessions were reported highly susceptible to stem borer (Chilo partellus S.), IS 1004, 4698, and 23216 were resistant to stem borer. Likewise, IS 4698 has been reported resistant to shoot fly (Atherigona soccata Rond.) (Sharma et al., 2003). These accessions with high Brix are ideal genetic resources for use in crop improvement programs. Sorghum researchers worldwide can obtain limited seed samples of accessions with high Brix content from the ICRISAT genebank for research purposes through a Standard Materials Transfer Agreement.

\section{CONCLUSIONS}

Sweet sorghum has gained importance worldwide as a bioenergy crop, particularly in countries in Asia, Africa, and the Americas. In this study, sorghum mini core collection was evaluated for stalk sugar and for phenological and agronomic traits under irrigated and drought stress conditions. Seven accessions with significantly greater Brix than the agronomically superior control (IS 33844) were identified, but these turned out to have lower yields. Four accessions significantly outyielded the control, IS 33844, but with similar Brix content. These accessions formed two distinct groups, clearly separating low-yielding accessions with high Brix from those having high yield and similar Brix to that of IS 33844. Some of these high Brix accessions were also reported to have resistance to leaf diseases and insect pests and therefore are ideal genetic resources for use in cultivar development for bioenergy traits in sorghum.

\section{References}

AgriFuels Limited. 2012. List of known sweet sorghum renewable energy projects. Agri-Fuels Ltd., Connecticut Center for Advanced Technol (CCAT). East Hartford, CT. www.agrifuels.com.au/OtherSweetSorghumBiofuelsProjects.htm (accessed 6 Jan. 2014).

Almodares, A., and M.R. Hadi. 2009. Production of bioethanol from sweet sorghum: A review. African J. Agric. Res. 4:772-780.

Almodares, A., R.H. Hotjatabady, and E. Mirniam. 2013. Effects of drought stress on biomass and carbohydrate contents of two sweet sorghum cultivars. J. Environ. Biol. 34:585-589.

Almodares, A., R. Taheri, and S. Adeli. 2008. Stalk yield and carbohydrate composition of sweet sorghum [Sorghum bicolor (L.) Moench] cultivars and lines at different growth stages. J. Malesian Appl. Biol. 37:31-36.

Assefa, Y., S.A. Staggenborg, and V.P.V. Prasad. 2010. Grain sorghum requirement and responses to drought stress: A review. Plant Manage. 9. doi:10.1094/CM-2010-1109-01-RV.

Bang, J.K., Y.B. Kim, S.S. Nam, S.H. Ahn, and S.J. Suh. 2009a. Variation of major characters in sweet sorghum germplasm for bioethanol. J. Korean Soc. Int. Agric. 21:189-192.

Bang, J.K., S.S. Nam, S.H. Ahn, and S.J. Suh. 2009b. Current research on sweet sorghum (Sorghum bicolor L. Moench) to bioethanol in China. J. Korean Soc. Int. Agric. 21:183-188. 
Billot, C., P. Ramu, S. Bouchet, J. Chantereau, M. Deu, L. Gardes, J.-L. Noyer, J.-F. Rami, R. Rivallan, Y. Li, P. Lu, T. Wang, R.T. Folkertsma, E. Arnaud, H.D. Upadhyaya, J.-C. Glaszmann, and C.T. Thomas. 2013. Massive sorghum collection genotyped with SSR markers to enhance use of global genetic resources. PLoS ONE 8:e59714. doi:10.1371/journal.pone.0059714

Blum, A. 2004. Sorghum physiology. In: H.T. Nguyen and A. Blum, editors, Physiology and Biotechnology Integration for Plant Breeding. Marcel Dekker, New York. p. 141-223.

Choi, Y.-H., Y.-H. Moon, S.-H. Ahn, Y.-M. Yoon, Y.-L. Cha, B.-C. Koo, K.-G. Park, H.-S. Han, and W.-S. Kim. 2012. Characteristics of sweet sorghum germplasm for bioethanol production in reclaimed soil. Korean J. Crop Sci. 57:384-388. doi:10.7740/ kjcs.2012.57.4.384

Codesido, V., R. Vacas, B. Macarulla, M.P. Gracia, and E. Igartua. 2013. Agronomic and digital phenotyping evaluation of sweet sorghum public varieties and $F_{1}$ hybrids with potential for ethanol production in Spain. Maydica 58:42-53.

de Roover, J., K. Vandenbranden, A.V. Laere, and W. Van den Ende. 2000. Drought induces fructan synthesis and 1-SST (sucrose:sucrose fructosyltransferase) in roots and leaves of chicory seedlings (Cichorium intybus L.). Planta 210:808-814. doi:10.1007/s004250050683

Dwivedi, S.L., K.L. Sahrawat, H.D. Upadhyaya, and R. Ortiz. 2013. Food, nutrition and agrobiodiversity under global climate change. Adv. Agron. 120:1-128. doi:10.1016/B978-0-12-407686-0.00001-4

Dwivedi, S.L., H.D. Upadhyaya, P. Subudhi, C. Gehring, V. Bajic, and R. Ortiz. 2010. Enhancing abiotic stress tolerance in cereals through breeding and transgenic interventions. Plant Breed. Rev. 33:31-114.

Eaton, F.M., and D.R. Ergle. 1948. Carbohydrate accumulation in the cotton plants at low moisture levels. Plant Physiol. 23:169-187. doi:10.1104/pp.23.2.169

El-Swaify, S.A., P. Pathak, T.J. Rigo, and S. Singh. 1985. Soil management for optimized productivity under rainfed conditions in the semi-arid tropics. Adv. Soil Sci. 1:1-64. doi:10.1007/978-1-46125046-3_1

Erickson, J.E., Z.R. Helsel, K.R. Woodard, J.M.B. Vendramini, Y. Wang, L.E. Sollenberger, and R.S. Gilbert. 2011. Planting date affects biomass and Brix of sweet sorghum grown for biofuels across Florida. Agron. J. 103:1827-1833. doi:10.2134/agronj2011.0176

EU. 2009. Directive 2009/28/EC of the European Parliament and of the Council of 23 April 2009 on the promotion of the use of energy from renewable sources and amending and subsequently repealing Directives 2001/77/EC and 2003/30/EC.

Frankel, O.H. 1984. Genetic perspective of germplasm conservation. In: W. Arber, K. Limensee, W.J. Peacock, and P. Stralinger, editors, Genetic manipulations: Impact on man and society. Cambridge Univ. Press, Cambridge, UK. p. 161-170.

Graham, J., V. Godoy, and T.T. Tesso. 2013. Analysis of juice yield, sugar content, and biomass accumulation in sorghum. Crop Sci. 53:1288-1297. doi:10.2135/cropsci2012.04.0217

Gutjahr, S., M. Vaksmann, M. Dingkuhn, K. Thera, G. Trouche, S. Braconnier, and D. Luquet. 2013. Grain, sugar and biomass accumulation in tropical sorghums. I. Trade-offs and effects of phenological plasticity. Funct. Plant Biol. 10.1071/FP12269.

Hagio, K., S. Sekimura, K. Ohta, and R. Meguro. 1987. Changes in heading times and sugar percentage (Brix\%) of sweet sorghum cultivars (Sorghum bicolor M.) in northern district of Japan. Tohoku Agric. Res. 40:197-198.

IBPGR and ICRISAT. 1993. Descriptors for Sorghum [Sorghum bicolor (L.) Moench]. Int. board for plant genetic resources, Rome, Italy. Int. Crops. Res. Inst. for the Semi-Arid Tropics, Patancheru, India.

Kameli, A., and D.M. Lösel. 1993. Carbohydrates and water status in wheat plants under water stress. New Phytol. 125:609-614. doi:10.1111/j.1469-8137.1993.tb03910.x
Kawahigashi, H., S. Kasuga, H. Okuizumi, S. Hiradate, and J. Yonemaru. 2013. Evaluation of brix and sugar content in stem juice from sorghum varieties. Grassland Sci. 59:11-19. doi:10.1111/ grs.12006

Kebede, H., P.K. Subudhi, D.T. Rosenow, and H.T. Nguyen. 2001. Quantitative trait loci influencing drought tolerance in grain sorghum (Sorghum bicolor L. Moench). Theor. Appl. Genet. 103:266276. doi:10.1007/s001220100541

Keuls, M. 1952. The use of "Studentized range" in connection with an analysis of variance. Euphytica 1:112-122. doi:10.1007/ BF01908269

Kholová, J., G. McLean, V. Vadez, P. Craufurd, and G.L. Hammer. 2012. Drought stress characterization of postrainy (rabi) sorghum in India. Field Crops Res. 141:38-46. doi:10.1016/j.fcr.2012.10.020

Kumar, A.A., B.V.S. Reddy, H.C. Sharma, C.T. Hash, P. Srinivasa Rao, B. Ramaiah, and P.S. Reddy. 2011. Recent advances in sorghum genetic enhancement research at ICRISAT. Am. J. Plant Sci. 2:589-600. doi:10.4236/ajps.2011.24070

Makanda, I., J. Derera, P. Tongoona, and J. Sibiya. 2012. Genetic and GGE biplot analyses of sorghum germplasm for stem sugar traits in Southern Africa. African J. Agric. Res. 72:212-223.

Makanda, I., P. Tongoona, and J. Derera. 2009. Quantification of genotypic variability for stem sugar accumulation and associated traits in new sorghum varieties. Afr. Crop Sci. Conf. Proc. 9:391-398.

Massacci, A., A. Battistelli, and F. Loreto. 1996. Effect of drought stress on photosynthetic characteristics, growth and sugar accumulation of field-grown sweet sorghum. Aust. J. Plant Physiol. 23:331-340. doi:10.1071/PP9960331

Méndez, A.M., D. Castillo, A. del Pozo, I. Matus, and R. Morcuende. 2011. Differences in stem soluble carbohydrate contents among recombinant chromosome substitution lines (RCSLs) of barley under drought in a Mediterranean-type environment. Agron. Res. 9:433-438.

Miri, K., and D.S. Rana. 2012. Evaluation of sweet sorghum (Sorghum bicolor) genotypes for biomass, sugar and ethanol production under different levels of nitrogen. Indian J. Agric. Sci. 82:195-200.

Mohammadkhani, N., and R. Heidari. 2008. Drought-induced accumulation of soluble sugars and proline in two maize varieties. World Appl. Sci. J. 3:448-453.

Murray, S.C., W.L. Rooney, S.E. Mitchell, A. Sharma, P.E. Klein, J.E. Mullet, and S. Kresovich. 2008. Genetic improvement of sorghum as a biofuel feedstock: II. QTL for stem and leaf structural carbohydrates. Crop Sci. 48:2180-2193. doi:10.2135/cropsci2008.01.0068

Newman, D. 1939. The distribution of range in samples from a normal population expressed in terms of an independent estimate of standard deviation. Biometrika 31:20-30. doi:10.1093/ biomet/31.1-2.20

Pavli, O.I., C.E. Vlachos, C. Kalloniati, E. Flemetakis, and G.N. Skaracis. 2013. Metabolite profiling reveals the effect of drought on sorghum (Sorghum bicolor L. Moench) metabolism. Plant Omics J. 6:371-376.

Perrier, X., A. Flori, and F. Bonnot. 2003. Data analysis methods. In: P. Hamon, M. Seguin, X. Perrier, and J.C. Glaszmann, editors, Genetic diversity of tropical plants. Enfields. Science Publishers, Montpellier, France. p. 43-76.

Praj Industries Limited. 2012. Sweet sorghum to ethanol. Technology, plant and machinery, Praj House, Bavdhan, Pune, India. www. praj.net/media/sweetsorghum.pdf (accessed 6 Jan. 2014).

Prasad, S., A. Singh, N. Jain, and H.C. Hoshi. 2007. Ethanol production from sweet sorghum syrup for utilization as automotive fuel in India. Energy Fuels 21:2415-2420. doi:10.1021/ef060328z

Ramel, F., C. Sulmon, G. Gouesbet, and I. Couée. 2009. Natural variation reveals relationships between pre-stress carbohydrate nutritional status and subsequent responses to xenobiotic and oxidative 
stress in Arabidopsis thaliana. Ann. Bot. (Lond.) 104:1323-1337. doi:10.1093/aob/mcp243

Rao, S.S., J.V. Patil, P.V.V. Prasad, D.C.S. Reddy, J.S. Mishra, A.V. Umakanth, B.V.S. Reddy, and A.A. Kumar. 2013. Sweet sorghum planting effects on stalk yield and sugar quality in semiarid tropical environment. Agron. J. 105:1458-1465. doi:10.2134/ agronj2013.0156

Reddy, B.V.S., S. Ramesh, S.T. Borikar, and H. Sahib. 2007. ICRISAT-Indian NARS partnership sorghum improvement research: Strategies and impacts. Curr. Sci. 92:909-915.

Reddy, B.V.S., S. Ramesh, P.S. Reddy, B. Ramaiah, P.M. Salimath, and K. Rajashekar. 2005. Sweet sorghum-A potential alternative raw material for bioethanol and bioenergy. Int. Sorghum Millet Newsl. 46:79-86.

Reddy, B.V.S., and P.S. Reddy. 2003. Sweet sorghum: Characteristics and potential. Int. Sorghum Millet Newsl. 44:26-28.

Renewable Fuels Association. 2012. Statistics. Renewable Fuels Association, Washington, DC. http://www.ethanolrfa.org/pages/ statistics (accessed 15 Nov. 2013).

Rooney, W.L., J. Blumenthal, B. Bean, and J.E. Mullet. 2007. Designing sorghum as dedicated bioenergy feedstock. Biofuels Bioprod. Biorefin. 1:147-157. doi:10.1002/bbb.15

Rosenow, D.T., and L.E. Clark. 1995. Drought and lodging resistance for a quality sorghum crop. Proceedings of the 50th Annual Corn and Sorghum Industry Research Conference, 6-7 Dec. 1995. American Seed Trade Association, Chicago, IL. p. 82-97.

Salazar, A.M., J.A.M. Tarun, C.B. Pascual, M. Caasi-Lit, P.J.A. Santos, C.E. Reaño, B.V.S. Reddy, and A.A. Kumar. 2009. Sweet sorghum hybrid parents improvement for bioethanol production for sustainable energy security in the Philippines. Philippines J. Crop Sci. 34 (Supplement no. 1):95 (abstract).

Schittenhelm, S., and S. Schroetter. 2014. Comparison of drought tolerance of maize, sweet sorghum and sorghum-Sudangrass hybrids. J. Agron. Crop Sci. 200:46-53. doi:10.1111/jac.12039

Seifers, D.L., R. Perumal, and C.R. Little. 2012. New sources of resistance in sorghum (Sorghum bicolor) germplasm are effective against a diverse array of Potyvirus spp. Plant Dis. 96:1775-1779. doi:10.1094/PDIS-03-12-0224-RE

Sharma, H.C. 1997. Host-plant resistance to shoot fly and spotted stem borer in sorghum. In: Strengthening sorghum research collaboration in Asia: Report of the Asian Sorghum Scientist's Meeting, Suphan Buri, Thailand, 18-21 Nov. 1997.

Sharma, H.C., S.L. Taneja, N. Kameswara Rao, and K.E. Prasada Rao. 2003. Evaluation of sorghum germplasm for resistance to insect pests. Information Bulletin no. 63. Patancheru 502 324. ICRISAT, Andhra Pradesh, India.

Sharma, R., H.D. Upadhyaya, S.V. Manjunath, V.P. Rao, and R.P. Thakur. 2012. Resistance to foliar diseases in a mini core collection of sorghum germplasm. Plant Dis. 96:1629-1633. doi:10.1094/ PDIS-10-11-0875-RE

Srinivasa Rao, P., S.S. Rao, N. Seetharama, A.V. Umakanth, P.S. Reddy, B.V.S. Reddy, and C.L.L. Gowda. 2009. Sweet sorghum for biofuel and strategies for its improvement. Inf. Bull. No. 77. Patancheru 502324. International Crops Research Institute for the Semi-Arid Tropics, Andhra Pradesh, India. p. 80.
Tari, I., G. Laskay, Z. Takács, and P. Poór. 2013. Response of sorghum to abiotic stresses: A review. J. Agron. Crop Sci. 199:264-274. doi:10.1111/jac.12017

Tata Chemicals Limited. 2012. Biofuels. TataChemicals, Fort, Mumbai. www.tatachemicals.com/products/biofuels.htm (accessed 6 Jan. 2014).

Thakur, R.P., V.P. Rao, B.M. Wu, K.V. Subbarao, K. Mathur, H.C. Tailor, U.S. Khushwaha, R.R. Dwivedi, R. Krishnawamy, R.V. Hiremath, and S. Indira. 2007. Genetic resistance to foliar anthracnose in sorghum and pathogenic variability in Colletotrichum graminicola. Indian Phytopathol. 60:13-23.

Tsuchihashi, N. 2004. Cultivation of sweet sorghum (Sorghum bicolor (L.) Moench) and determination of its harvest time to make use as the raw material for fermentation, practiced during rainy season in dryland of Indonesia. Plant Prod. Sci. 7:442-448. doi:10.1626/ pps.7.442

Tsuchihashi, N., and Y. Goto. 2008. Year-round cultivation of sweet sorghum [Sorghum bicolor (L.) Moench] through a combination of seed and ratoon cropping in Indonesian Savanna. Plant Prod. Sci. 11:377-384. doi:10.1626/pps.11.377

Tuinstra, M.R., E.M. Grote, P.B. Goldsbrough, and G. Ejeta. 1996. Identification of quantitativetrait loci associated with post-flowering drought tolerance in sorghum. Crop Sci. 36:1337-1344. doi:10.2135/cropsci1996.0011183X003600050043x

Upadhyaya, H.D., and R. Ortiz. 2001. A mini core collection for capturing diversity and promoting utilization of chickpea genetic resources in crop improvement. Theor. Appl. Genet. 102:12921298. doi:10.1007/s00122-001-0556-y

Upadhyaya, H.D., R.P.S. Pundir, S.L. Dwivedi, and C.L.L. Gowda. 2009a. Mini core collections for efficient utilization of plant genetic resources in crop improvement programs. Information Bulletin No. 8. International Crops Research Institute for the Semi-Arid Tropics, Patancheru 502324, Andhra Pradesh, India. p. 52 .

Upadhyaya, H.D., R.P.S. Pundir, S.L. Dwivedi, C.L.L. Gowda, V.G. Reddy, and S. Singh. 2009b. Developing a mini core collection of sorghum for diversified utilization of germplasm. Crop Sci. 49:1769-1780. doi:10.2135/cropsci2009.01.0014

Vassiliev, I.M., and M.G. Vassiliev. 1936. Changes in carbohydrate content of wheat plant during the process of hardening for drought resistance. Plant Physiol. 11:115-125. doi:10.1104/pp.11.1.115

V.S.N. International. 2013. GenStat software for windows. Release 14.1. VSNL International Ltd., Hemel Hempstead. Hertfordshire, UK.

Zegada-Lizarazu, W., H.W. Elbersen, S.L. Cosentino, A. Zatta, E. Alexopoulou, and A. Monti. 2010. Agronomic aspects of future energy crops in Europe. Biofuels Bioprod. Biorefin. 4:674-691. doi:10.1002/bbb. 242

Zou, G., S. Yan, G. Zhai, Z. Zhang, J. Zou, and Y. Tao. 2011. Genetic variability and correlation of stalk yield-related traits and sugar concentration of stalk juice in a sweet sorghum (Sorghum bicolor L. Moench) population. Austr. J. Crop Sci. 5:1232-1238. 